\title{
Simplified Variational Principles for non-Barotropic Magnetohydrodynamics
}

\author{
Asher Yahalom ${ }^{a}$ \\ ${ }^{a}$ Ariel University, Ariel 40700, Israel \\ e-mail: asya@ariel.ac.il;
}

May 24, 2022

\begin{abstract}
Variational principles for magnetohydrodynamics were introduced by previous authors both in Lagrangian and Eulerian form. In this paper we introduce simpler Eulerian variational principles from which all the relevant equations of non-barotropic magnetohydrodynamics can be derived. The variational principle is given in terms of five independent functions for non-stationary barotropic flows. This is less then the eight variables which appear in the standard equations of barotropic magnetohydrodynamics which are the magnetic field $\vec{B}$ the velocity field $\vec{v}$, the entropy $s$ and the density $\rho$.
\end{abstract}

Keywords: Magnetohydrodynamics, Variational principles

PACS number(s): 47.65.+a

\section{Introduction}

Variational principles for magnetohydrodynamics were introduced by previous authors both in Lagrangian and Eulerian form. Sturrock 1 has discussed in his book a Lagrangian variational formalism for magnetohydrodynamics. Vladimirov and Moffatt [2 in a series of papers have discussed an Eulerian variational principle for incompressible magnetohydrodynamics. However, their variational principle contained three more functions in addition to the seven variables which appear in the standard equations of incompressible magnetohydrodynamics which are the magnetic field $\vec{B}$ the velocity field $\vec{v}$ and the pressure $P$. Kats [3] has generalized Moffatt's work for compressible non barotropic flows but without reducing the number of functions and the computational load. Moreover, Kats has shown that the variables he suggested can be utilized to describe the motion of arbitrary discontinuity surfaces [4, 5]. Sakurai [6] has introduced a two function Eulerian variational principle for force-free magnetohydrodynamics and used it as a basis of a numerical scheme, his method is 
discussed in a book by Sturrock [1]. A method of solving the equations for those two variables was introduced by Yang, Sturrock \& Antiochos [7. Yahalom \& Lynden-Bell [8] combined the Lagrangian of Sturrock [1] with the Lagrangian of Sakurai [6] to obtain an Eulerian Lagrangian principle for barotropic magnetohydrodynamics which will depend on only six functions. The variational derivative of this Lagrangian produced all the equations needed to describe barotropic magnetohydrodynamics without any additional constraints. The equations obtained resembled the equations of Frenkel, Levich \& Stilman [11] (see also [12]). Yahalom [9] have shown that for the barotropic case four functions will suffice. Moreover, it was shown that the cuts of some of those functions [10] are topological local conserved quantities.

Previous work was concerned only with barotropic magnetohydrodynamics. Variational principles of non barotropic magnetohydrodynamics can be found in the work of Bekenstein \& Oron [13] in terms of 15 functions and V.A. Kats 3 in terms of 20 functions. The author of this paper suspect that this number can be somewhat reduced. Moreover, A. V. Kats in a remarkable paper [14] (section IV,E) has shown that there is a large symmetry group (gauge freedom) associated with the choice of those functions, this implies that the number of degrees of freedom can be reduced. Here we will show that only five functions will suffice to describe non barotropic magnetohydrodynamics in the case that we enforce a Sakurai [6] representation for the magnetic field.

We anticipate applications of this study both to linear and non-linear stability analysis of known non barotropic magnetohydrodynamic configurations 20, 22 and for designing efficient numerical schemes for integrating the equations of fluid dynamics and magnetohydrodynamics [28, 29, 30, 31]. Another possible application is connected to obtaining new analytic solutions in terms of the variational variables [32].

The plan of this paper is as follows: First we introduce the standard notations and equations of non-barotropic magnetohydrodynamics. Next we introduce a generalization of the barotropic variational principle suitable for the non-barotropic case. Later we simplify the Eulerian variational principle and formulate it in terms of eight functions. Next we show how three variational variables can be integrated algebraically thus reducing the variational principle to five functions. We conclude by writing down the appropriate Hamiltonian for non-barotropic magnetohydrodynamics and writing the equivalent Hamilton's equations for this case.

\section{Standard formulation of non-barotropic mag- netohydrodynamics}

The standard set of equations solved for non-barotropic magnetohydrodynamics are given below:

$$
\frac{\partial \vec{B}}{\partial t}=\vec{\nabla} \times(\vec{v} \times \vec{B})
$$




$$
\begin{gathered}
\vec{\nabla} \cdot \vec{B}=0, \\
\frac{\partial \rho}{\partial t}+\vec{\nabla} \cdot(\rho \vec{v})=0, \\
\rho \frac{d \vec{v}}{d t}=\rho\left(\frac{\partial \vec{v}}{\partial t}+(\vec{v} \cdot \vec{\nabla}) \vec{v}\right)=-\vec{\nabla} p(\rho, s)+\frac{(\vec{\nabla} \times \vec{B}) \times \vec{B}}{4 \pi} . \\
\frac{d s}{d t}=0 .
\end{gathered}
$$

The following notations are utilized: $\frac{\partial}{\partial t}$ is the temporal derivative, $\frac{d}{d t}$ is the temporal material derivative and $\vec{\nabla}$ has its standard meaning in vector calculus. $\vec{B}$ is the magnetic field vector, $\vec{v}$ is the velocity field vector, $\rho$ is the fluid density and $s$ is the specific entropy. Finally $p(\rho, s)$ is the pressure which depends on the density and entropy (the non-barotropic case). The justification for those equations and the conditions under which they apply can be found in standard books on magnetohydrodynamics (see for example [1]). Equation (1) describes the fact that the magnetic field lines are moving with the fluid elements ("frozen" magnetic field lines), equation (21) describes the fact that the magnetic field is solenoidal, equation (3) describes the conservation of mass and equation (4) is the Euler equation for a fluid in which both pressure and Lorentz magnetic forces apply. The term:

$$
\vec{J}=\frac{\vec{\nabla} \times \vec{B}}{4 \pi},
$$

is the electric current density which is not connected to any mass flow. Equation (5) describes the fact that heat is not created (zero viscosity, zero resistivity) in ideal non-barotropic magnetohydrodynamics and is not conducted, thus only convection occurs. The number of independent variables for which one needs to solve is eight $(\vec{v}, \vec{B}, \rho, s)$ and the number of equations (1/3/4/5) is also eight.

Notice that equation (2) is a condition on the initial $\vec{B}$ field and is satisfied automatically for any other time due to equation (1).

\section{Variational principle of non-barotropic mag- netohydrodynamics}

In the following section we will generalize the approach of 8 for the nonbarotropic case. Consider the action:

$$
\begin{aligned}
A & \equiv \int \mathcal{L} d^{3} x d t \\
\mathcal{L} & \equiv \mathcal{L}_{1}+\mathcal{L}_{2} \\
\mathcal{L}_{1} & \equiv \rho\left(\frac{1}{2} \vec{v}^{2}-\varepsilon(\rho, s)\right)+\frac{\vec{B}^{2}}{8 \pi} \\
\mathcal{L}_{2} & \equiv \nu\left[\frac{\partial \rho}{\partial t}+\vec{\nabla} \cdot(\rho \vec{v})\right]-\rho \alpha \frac{d \chi}{d t}-\rho \beta \frac{d \eta}{d t}-\rho \sigma \frac{d s}{d t}-\frac{\vec{B}}{4 \pi} \cdot \vec{\nabla} \chi \times \vec{\nabla} \eta
\end{aligned}
$$


Obviously $\nu, \alpha, \beta, \sigma$ are Lagrange multipliers which were inserted in such a way that the variational principle will yield the following equations:

$$
\begin{aligned}
& \frac{\partial \rho}{\partial t}+\vec{\nabla} \cdot(\rho \vec{v})=0, \\
& \rho \frac{d \chi}{d t}=0, \\
& \rho \frac{d \eta}{d t}=0 . \\
& \rho \frac{d s}{d t}=0 .
\end{aligned}
$$

It is not assumed that $\nu, \alpha, \beta, \sigma$ are single valued. Provided $\rho$ is not null those are just the continuity equation (3), entropy conservation and the conditions that Sakurai's functions are comoving. Taking the variational derivative with respect to $\vec{B}$ we see that

$$
\vec{B}=\hat{\vec{B}} \equiv \vec{\nabla} \chi \times \vec{\nabla} \eta .
$$

Hence $\vec{B}$ is in Sakurai's form and satisfies equation (2). It can be easily shown that provided that $\vec{B}$ is in the form given in equation (9), and equations (8) are satisfied, then also equation (1) is satisfied.

For the time being we have showed that all the equations of non-barotropic magnetohydrodynamics can be obtained from the above variational principle except Euler's equations. We will now show that Euler's equations can be derived from the above variational principle as well. Let us take an arbitrary variational derivative of the above action with respect to $\vec{v}$, this will result in:

$\delta_{\vec{v}} A=\int d t\left\{\int d^{3} x d t \rho \delta \vec{v} \cdot[\vec{v}-\vec{\nabla} \nu-\alpha \vec{\nabla} \chi-\beta \vec{\nabla} \eta-\sigma \vec{\nabla} s]+\oint d \vec{S} \cdot \delta \vec{v} \rho \nu+\int d \vec{\Sigma} \cdot \delta \vec{v} \rho[\nu]\right\}$.

The integral $\oint d \vec{S} \cdot \delta \vec{v} \rho \nu$ vanishes in many physical scenarios. In the case of astrophysical flows this integral will vanish since $\rho=0$ on the flow boundary, in the case of a fluid contained in a vessel no flux boundary conditions $\delta \vec{v} \cdot \hat{n}=0$ are induced ( $\hat{n}$ is a unit vector normal to the boundary). The surface integral $\int d \vec{\Sigma}$ on the cut of $\nu$ vanishes in the case that $\nu$ is single valued and $[\nu]=0$. In the case that $\nu$ is not single valued only a Kutta type velocity perturbation [30 in which the velocity perturbation is parallel to the cut will cause the cut integral to vanish.

Provided that the surface integrals do vanish and that $\delta_{\vec{v}} A=0$ for an arbitrary velocity perturbation we see that $\vec{v}$ must have the following form:

$$
\vec{v}=\hat{\vec{v}} \equiv \vec{\nabla} \nu+\alpha \vec{\nabla} \chi+\beta \vec{\nabla} \eta+\sigma \vec{\nabla} s .
$$

The above equation is reminiscent of Clebsch representation in non magnetic fluids [33, 34]. Let us now take the variational derivative with respect to the density $\rho$ we obtain:

$$
\delta_{\rho} A=\int d^{3} x d t \delta \rho\left[\frac{1}{2} \vec{v}^{2}-w-\frac{\partial \nu}{\partial t}-\vec{v} \cdot \vec{\nabla} \nu\right]
$$




$$
+\int d t \oint d \vec{S} \cdot \vec{v} \delta \rho \nu+\int d t \int d \vec{\Sigma} \cdot \vec{v} \delta \rho[\nu]+\left.\int d^{3} x \nu \delta \rho\right|_{t_{0}} ^{t_{1}} .
$$

In which $w=\frac{\partial(\varepsilon \rho)}{\partial \rho}$ is the specific enthalpy. Hence provided that $\oint d \vec{S} \cdot \vec{v} \delta \rho \nu$ vanishes on the boundary of the domain and $\int d \vec{\Sigma} \cdot \vec{v} \delta \rho[\nu]$ vanishes on the cut of $\nu$ in the case that $\nu$ is not single valued 1 and in initial and final times the following equation must be satisfied:

$$
\frac{d \nu}{d t}=\frac{1}{2} \vec{v}^{2}-w
$$

Finally we have to calculate the variation with respect to both $\chi$ and $\eta$ this will lead us to the following results:

$$
\begin{aligned}
\delta_{\chi} A & =\int d^{3} x d t \delta \chi\left[\frac{\partial(\rho \alpha)}{\partial t}+\vec{\nabla} \cdot(\rho \alpha \vec{v})-\vec{\nabla} \eta \cdot \vec{J}\right]+\int d t \oint d \vec{S} \cdot\left[\frac{\vec{B}}{4 \pi} \times \vec{\nabla} \eta-\vec{v} \rho \alpha\right] \delta \chi \\
& +\int d t \int d \vec{\Sigma} \cdot\left[\frac{\vec{B}}{4 \pi} \times \vec{\nabla} \eta-\vec{v} \rho \alpha\right][\delta \chi]-\left.\int d^{3} x \rho \alpha \delta \chi\right|_{t_{0}} ^{t_{1}} \\
\delta_{\eta} A & =\int d^{3} x d t \delta \eta\left[\frac{\partial(\rho \beta)}{\partial t}+\vec{\nabla} \cdot(\rho \beta \vec{v})+\vec{\nabla} \chi \cdot \vec{J}\right]+\int d t \oint d \vec{S} \cdot\left[\vec{\nabla} \chi \times \frac{\vec{B}}{4 \pi}-\vec{v} \rho \beta\right] \delta \eta \\
& +\int d t \int d \vec{\Sigma} \cdot\left[\vec{\nabla} \chi \times \frac{\vec{B}}{4 \pi}-\vec{v} \rho \beta\right][\delta \eta]-\left.\int d^{3} x \rho \beta \delta \eta\right|_{t_{0}} ^{t_{1}} .
\end{aligned}
$$

Provided that the correct temporal and boundary conditions are met with respect to the variations $\delta \chi$ and $\delta \eta$ on the domain boundary and on the cuts in the case that some (or all) of the relevant functions are non single valued. we obtain the following set of equations:

$$
\frac{d \alpha}{d t}=\frac{\vec{\nabla} \eta \cdot \vec{J}}{\rho}, \quad \frac{d \beta}{d t}=-\frac{\vec{\nabla} \chi \cdot \vec{J}}{\rho},
$$

in which the continuity equation (3) was taken into account. By correct temporal conditions we mean that both $\delta \eta$ and $\delta \chi$ vanish at initial and final times. As for boundary conditions which are sufficient to make the boundary term vanish on can consider the case that the boundary is at infinity and both $\vec{B}$ and $\rho$ vanish. Another possibility is that the boundary is impermeable and perfectly conducting. A sufficient condition for the integral over the "cuts" to vanish is to use variations $\delta \eta$ and $\delta \chi$ which are single valued. It can be shown that $\chi$ can always be taken to be single valued, hence taking $\delta \chi$ to be single valued is no restriction at all. In some topologies $\eta$ is not single valued and in those cases a single valued restriction on $\delta \eta$ is sufficient to make the cut term null.

\footnotetext{
${ }^{1}$ Which entails either a Kutta type condition for the velocity or a vanishing density perturbation on the cut.
} 
Finally we take a variational derivative with respect to the entropy $s$ :

$$
\begin{aligned}
\delta_{s} A & =\int d^{3} x d t \delta s\left[\frac{\partial(\rho \sigma)}{\partial t}+\vec{\nabla} \cdot(\rho \sigma \vec{v})-\rho T\right]+\int d t \oint d \vec{S} \cdot \rho \sigma \vec{v} \delta s \\
& -\left.\int d^{3} x \rho \sigma \delta s\right|_{t_{0}} ^{t_{1}},
\end{aligned}
$$

in which the temperature is $T=\frac{\partial \varepsilon}{\partial s}$. We notice that according to equation (11) $\sigma$ is single valued and hence no cuts are needed. Taking into account the continuity equation (3) we obtain for locations in which the density $\rho$ is not null the result:

$$
\frac{d \sigma}{d t}=T
$$

provided that $\delta_{s} A$ vanished for an arbitrary $\delta s$.

\section{Euler's equations}

We shall now show that a velocity field given by equation (11), such that the equations for $\alpha, \beta, \chi, \eta, \nu, \sigma, s$ satisfy the corresponding equations (8]13]16]18) must satisfy Euler's equations. Let us calculate the material derivative of $\vec{v}$ :

$$
\frac{d \vec{v}}{d t}=\frac{d \vec{\nabla} \nu}{d t}+\frac{d \alpha}{d t} \vec{\nabla} \chi+\alpha \frac{d \vec{\nabla} \chi}{d t}+\frac{d \beta}{d t} \vec{\nabla} \eta+\beta \frac{d \vec{\nabla} \eta}{d t}+\frac{d \sigma}{d t} \vec{\nabla} s+\sigma \frac{d \vec{\nabla} s}{d t} .
$$

It can be easily shown that:

$$
\begin{aligned}
\frac{d \vec{\nabla} \nu}{d t} & =\vec{\nabla} \frac{d \nu}{d t}-\vec{\nabla} v_{k} \frac{\partial \nu}{\partial x_{k}}=\vec{\nabla}\left(\frac{1}{2} \vec{v}^{2}-w\right)-\vec{\nabla} v_{k} \frac{\partial \nu}{\partial x_{k}} \\
\frac{d \vec{\nabla} \eta}{d t} & =\vec{\nabla} \frac{d \eta}{d t}-\vec{\nabla} v_{k} \frac{\partial \eta}{\partial x_{k}}=-\vec{\nabla} v_{k} \frac{\partial \eta}{\partial x_{k}} \\
\frac{d \vec{\nabla} \chi}{d t} & =\vec{\nabla} \frac{d \chi}{d t}-\vec{\nabla} v_{k} \frac{\partial \chi}{\partial x_{k}}=-\vec{\nabla} v_{k} \frac{\partial \chi}{\partial x_{k}} \\
\frac{d \vec{\nabla} s}{d t} & =\vec{\nabla} \frac{d s}{d t}-\vec{\nabla} v_{k} \frac{\partial s}{\partial x_{k}}=-\vec{\nabla} v_{k} \frac{\partial s}{\partial x_{k}} .
\end{aligned}
$$

In which $x_{k}$ is a Cartesian coordinate and a summation convention is assumed. Inserting the result from equations (2018) into equation (19) yields:

$$
\begin{aligned}
\frac{d \vec{v}}{d t} & =-\vec{\nabla} v_{k}\left(\frac{\partial \nu}{\partial x_{k}}+\alpha \frac{\partial \chi}{\partial x_{k}}+\beta \frac{\partial \eta}{\partial x_{k}}+\sigma \frac{\partial s}{\partial x_{k}}\right)+\vec{\nabla}\left(\frac{1}{2} \vec{v}^{2}-w\right)+T \vec{\nabla} s \\
& +\frac{1}{\rho}((\vec{\nabla} \eta \cdot \vec{J}) \vec{\nabla} \chi-(\vec{\nabla} \chi \cdot \vec{J}) \vec{\nabla} \eta) \\
& =-\vec{\nabla} v_{k} v_{k}+\vec{\nabla}\left(\frac{1}{2} \vec{v}^{2}-w\right)+T \vec{\nabla} s+\frac{1}{\rho} \vec{J} \times(\vec{\nabla} \chi \times \vec{\nabla} \eta) \\
& =-\frac{\vec{\nabla} p}{\rho}+\frac{1}{\rho} \vec{J} \times \vec{B} .
\end{aligned}
$$


In which we have used both equation (11) and equation (9) in the above derivation. This of course proves that the non-barotropic Euler equations can be derived from the action given in equation (77) and hence all the equations of non-barotropic magnetohydrodynamics can be derived from the above action without restricting the variations in any way except on the relevant boundaries and cuts.

\section{Simplified action}

The reader of this paper might argue here that the paper is misleading. The author has declared that he is going to present a simplified action for nonbarotropic magnetohydrodynamics instead he added six more functions $\alpha, \beta, \chi,-$ $\eta, \nu, \sigma$ to the standard set $\vec{B}, \vec{v}, \rho, s$. In the following I will show that this is not so and the action given in equation (7) in a form suitable for a pedagogic presentation can indeed be simplified. It is easy to show that the Lagrangian density appearing in equation (7) can be written in the form:

$$
\begin{aligned}
\mathcal{L} & =-\rho\left[\frac{\partial \nu}{\partial t}+\alpha \frac{\partial \chi}{\partial t}+\beta \frac{\partial \eta}{\partial t}+\sigma \frac{\partial s}{\partial t}+\varepsilon(\rho, s)\right]+\frac{1}{2} \rho\left[(\vec{v}-\hat{\vec{v}})^{2}-(\hat{\vec{v}})^{2}\right] \\
& +\frac{1}{8 \pi}\left[(\vec{B}-\hat{\vec{B}})^{2}-(\hat{\vec{B}})^{2}\right]+\frac{\partial(\nu \rho)}{\partial t}+\vec{\nabla} \cdot(\nu \rho \vec{v}) .
\end{aligned}
$$

In which $\hat{\vec{v}}$ is a shorthand notation for $\vec{\nabla} \nu+\alpha \vec{\nabla} \chi+\beta \vec{\nabla} \eta+\sigma \vec{\nabla} s$ (see equation (111)) and $\hat{\vec{B}}$ is a shorthand notation for $\vec{\nabla} \chi \times \vec{\nabla} \eta$ (see equation (9)). Thus $\mathcal{L}$ has four contributions:

$$
\begin{aligned}
& \mathcal{L}=\hat{\mathcal{L}}+\mathcal{L}_{\vec{v}}+\mathcal{L}_{\vec{B}}+\mathcal{L}_{\text {boundary }}, \\
& \hat{\mathcal{L}} \equiv-\rho\left[\frac{\partial \nu}{\partial t}+\alpha \frac{\partial \chi}{\partial t}+\beta \frac{\partial \eta}{\partial t}+\sigma \frac{\partial s}{\partial t}+\varepsilon(\rho, s)+\frac{1}{2}(\vec{\nabla} \nu+\alpha \vec{\nabla} \chi+\beta \vec{\nabla} \eta+\sigma \vec{\nabla} s)^{2}\right] \\
& \quad-\frac{1}{8 \pi}(\vec{\nabla} \chi \times \vec{\nabla} \eta)^{2} \\
& \mathcal{L}_{\vec{v}} \equiv \frac{1}{2} \rho(\vec{v}-\hat{\vec{v}})^{2}, \\
& \mathcal{L}_{\vec{B}} \equiv \frac{1}{8 \pi}(\vec{B}-\hat{\vec{B}})^{2}, \\
& \mathcal{L}_{\text {boundary }} \equiv \frac{\partial(\nu \rho)}{\partial t}+\vec{\nabla} \cdot(\nu \rho \vec{v}) .
\end{aligned}
$$

The only term containing $\vec{v}$ is $\mathcal{L}_{\vec{v}}$, it can easily be seen that this term will lead, after we nullify the variational derivative with respect to $\vec{v}$, to equation (11) but will otherwise have no contribution to other variational derivatives. Similarly the only term containing $\vec{B}$ is $\mathcal{L}_{\vec{B}}$ and it can easily be seen that this term will lead, after we nullify the variational derivative, to equation (9) but will

\footnotetext{
${ }^{2} \mathcal{L}_{\text {boundary }}$ also depends on $\vec{v}$ but being a boundary term is space and time it does not contribute to the derived equations
} 
have no contribution to other variational derivatives. Also notice that the term $\mathcal{L}_{\text {boundary }}$ contains only complete partial derivatives and thus can not contribute to the equations although it can change the boundary conditions. Hence we see that equations (8), equation (13), equations (16) and equation (18) can be derived using the Lagrangian density:

$$
\begin{aligned}
& \hat{\mathcal{L}}[\alpha, \beta, \chi, \eta, \nu, \rho, \sigma, s]=-\rho\left[\frac{\partial \nu}{\partial t}+\alpha \frac{\partial \chi}{\partial t}+\beta \frac{\partial \eta}{\partial t}+\sigma \frac{\partial s}{\partial t}\right. \\
& \left.+\varepsilon(\rho, s)+\frac{1}{2}(\vec{\nabla} \nu+\alpha \vec{\nabla} \chi+\beta \vec{\nabla} \eta+\sigma \vec{\nabla} s)^{2}\right]-\frac{1}{8 \pi}(\vec{\nabla} \chi \times \vec{\nabla} \eta)^{2}
\end{aligned}
$$

in which $\hat{\vec{v}}$ replaces $\vec{v}$ and $\hat{\vec{B}}$ replaces $\vec{B}$ in the relevant equations. Furthermore, after integrating the eight equations (81316 18) we can insert the potentials $\alpha, \beta, \chi, \eta, \nu, \sigma, s$ into equations (11) and (9) to obtain the physical quantities $\vec{v}$ and $\vec{B}$. Hence, the general non-barotropic magnetohydrodynamic problem is reduced from eight equations (13/45) and the additional constraint (2) to a problem of eight first order (in the temporal derivative) unconstrained equations. Moreover, the entire set of equations can be derived from the Lagrangian density $\hat{\mathcal{L}}$.

\section{Further Simplification}

\subsection{Elimination of Variables}

Let us now look at the three last three equations of (8) . Those describe three comoving quantities which can be written in terms of the generalized Clebsch form given in equation (11) as follows:

$$
\begin{aligned}
& \frac{\partial \chi}{\partial t}+(\vec{\nabla} \nu+\alpha \vec{\nabla} \chi+\beta \vec{\nabla} \eta+\sigma \vec{\nabla} s) \cdot \vec{\nabla} \chi=0 \\
& \frac{\partial \eta}{\partial t}+(\vec{\nabla} \nu+\alpha \vec{\nabla} \chi+\beta \vec{\nabla} \eta+\sigma \vec{\nabla} s) \cdot \vec{\nabla} \eta=0 \\
& \frac{\partial s}{\partial t}+(\vec{\nabla} \nu+\alpha \vec{\nabla} \chi+\beta \vec{\nabla} \eta+\sigma \vec{\nabla} s) \cdot \vec{\nabla} s=0
\end{aligned}
$$

Those are algebraic equations for $\alpha, \beta, \sigma$. Which can be solved such that $\alpha, \beta, \sigma$ can be written as functionals of $\chi, \eta, \nu, s$, resulting eventually in the description of non-barotropic magnetohydrodynamics in terms of five functions: $\nu, \rho, \chi, \eta, s$. Let us introduce the notation:

$$
\alpha_{i} \equiv(\alpha, \beta, \sigma), \quad \chi_{i} \equiv(\chi, \eta, s), \quad k_{i} \equiv-\frac{\partial \chi_{i}}{\partial t}-\vec{\nabla} \nu \cdot \vec{\nabla} \chi_{i}, \quad i \in(1,2,3)
$$

In terms of the above notation equation (25) takes the form:

$$
k_{i}=\alpha_{j} \vec{\nabla} \chi_{i} \cdot \vec{\nabla} \chi_{j}, \quad j \in(1,2,3)
$$


in which the Einstein summation convention is assumed. Let us define the matrix:

$$
A_{i j} \equiv \vec{\nabla} \chi_{i} \cdot \vec{\nabla} \chi_{j}
$$

obviously this matrix is symmetric since $A_{i j}=A_{j i}$. Hence equation (27) takes the form:

$$
k_{i}=A_{i j} \alpha_{j}, \quad j \in(1,2,3)
$$

Provided that the matrix $A_{i j}$ is not singular it has an inverse $A_{i j}^{-1}$ which can be written as:

$$
A_{i j}^{-1}=|A|^{-1}\left(\begin{array}{ccc}
A_{22} A_{33}-A_{23}^{2} & A_{13} A_{23}-A_{12} A_{33} & A_{12} A_{23}-A_{13} A_{22} \\
A_{13} A_{23}-A_{12} A_{33} & A_{11} A_{33}-A_{13}^{2} & A_{12} A_{13}-A_{11} A_{23} \\
A_{12} A_{23}-A_{13} A_{22} & A_{12} A_{13}-A_{11} A_{23} & A_{11} A_{22}-A_{12}^{2}
\end{array}\right)
$$

In which the determinant $|A|$ is given by the following equation:

$$
|A|=A_{11} A_{22} A_{33}-A_{11} A_{23}^{2}-A_{22} A_{13}^{2}-A_{33} A_{12}^{2}+2 A_{12} A_{13} A_{23}
$$

In terms of the above equations the $\alpha_{i}$ 's can be calculated as functionals of $\chi_{i}, \nu$ as follows:

$$
\alpha_{i}\left[\chi_{i}, \nu\right]=A_{i j}^{-1} k_{j}
$$

The velocity equation (11) can now be written as:

$$
\vec{v}=\vec{\nabla} \nu+\alpha_{i} \vec{\nabla} \chi_{i}=\vec{\nabla} \nu+A_{i j}^{-1} k_{j} \vec{\nabla} \chi_{i}=\vec{\nabla} \nu-A_{i j}^{-1} \vec{\nabla} \chi_{i}\left(\frac{\partial \chi_{j}}{\partial t}+\vec{\nabla} \nu \cdot \vec{\nabla} \chi_{j}\right)
$$

Provided that the $\chi_{i}$ is a coordinate basis in three dimensions, we may write:

$$
\vec{\nabla} \nu=\vec{\nabla} \chi_{n} \frac{\partial \nu}{\partial \chi_{n}}, \quad n \in(1,2,3)
$$

Inserting equation (34) into equation (33) we obtain:

$$
\begin{aligned}
\vec{v} & =-A_{i j}^{-1} \vec{\nabla} \chi_{i} \frac{\partial \chi_{j}}{\partial t}+\vec{\nabla} \nu-A_{i j}^{-1} \vec{\nabla} \chi_{i} \frac{\partial \nu}{\partial \chi_{n}} \vec{\nabla} \chi_{n} \cdot \vec{\nabla} \chi_{j} \\
& =-A_{i j}^{-1} \vec{\nabla} \chi_{i} \frac{\partial \chi_{j}}{\partial t}+\vec{\nabla} \nu-A_{i j}^{-1} A_{j n} \vec{\nabla} \chi_{i} \frac{\partial \nu}{\partial \chi_{n}} \\
& =-A_{i j}^{-1} \vec{\nabla} \chi_{i} \frac{\partial \chi_{j}}{\partial t}+\vec{\nabla} \nu-\delta_{i n} \vec{\nabla} \chi_{i} \frac{\partial \nu}{\partial \chi_{n}} \\
& =-A_{i j}^{-1} \vec{\nabla} \chi_{i} \frac{\partial \chi_{j}}{\partial t}+\vec{\nabla} \nu-\vec{\nabla} \chi_{n} \frac{\partial \nu}{\partial \chi_{n}} \\
& =-A_{i j}^{-1} \vec{\nabla} \chi_{i} \frac{\partial \chi_{j}}{\partial t}
\end{aligned}
$$

in the above $\delta_{i n}$ is a Kronecker delta. Thus the velocity $\vec{v}\left[\chi_{i}\right]$ is a functional of $\chi_{i}$ only and is independent of $\nu$. 


\subsection{Lagrangian Density and Variational Analysis}

Let us now rewrite the Lagrangian density $\hat{\mathcal{L}}\left[\chi_{i}, \nu, \rho\right]$ given in equation (24) in terms of the new variables:

$$
\hat{\mathcal{L}}\left[\chi_{i}, \nu, \rho\right]=-\rho\left[\frac{\partial \nu}{\partial t}+\alpha_{k}\left[\chi_{i}, n u\right] \frac{\partial \chi_{k}}{\partial t}+\varepsilon\left(\rho, \chi_{3}\right)+\frac{1}{2} \vec{v}\left[\chi_{i}\right]^{2}\right]-\frac{1}{8 \pi}\left(\vec{\nabla} \chi_{1} \times \vec{\nabla} \chi_{2}\right)^{2}
$$

Let us calculate the variational derivative of $\hat{\mathcal{L}}\left[\chi_{i}, \nu, \rho\right]$ with respect to $\chi_{i}$ this will result in:

$$
\delta_{\chi_{i}} \hat{\mathcal{L}}=-\rho\left[\delta_{\chi_{i}} \alpha_{k} \frac{\partial \chi_{k}}{\partial t}+\alpha_{\underline{i}} \frac{\partial \delta \chi_{\underline{i}}}{\partial t}+\delta_{\chi_{i}} \varepsilon\left(\rho, \chi_{3}\right)+\delta_{\chi_{i}} \vec{v} \cdot \vec{v}\right]-\frac{\vec{B}}{4 \pi} \cdot \delta_{\chi_{i}}\left(\vec{\nabla} \chi_{1} \times \vec{\nabla} \chi_{2}\right)
$$

in which the summation convention is not applied if the index is underlined. However, due to equation (33) we may write:

$$
\delta_{\chi_{i}} \vec{v}=\delta_{\chi_{i}} \alpha_{k} \vec{\nabla} \chi_{k}+\alpha_{\underline{i}} \vec{\nabla} \delta \chi_{\underline{i}}
$$

Inserting equation (38) into equation (37) and rearranging the terms we obtain:

$$
\begin{aligned}
\delta_{\chi_{i}} \hat{\mathcal{L}} & =-\rho\left[\delta_{\chi_{i}} \alpha_{k}\left(\frac{\partial \chi_{k}}{\partial t}+\vec{v} \cdot \vec{\nabla} \chi_{k}\right)+\alpha_{\underline{i}}\left(\frac{\partial \delta \chi_{\underline{i}}}{\partial t}+\vec{v} \cdot \vec{\nabla} \delta \chi_{\underline{i}}\right)+\delta_{\chi_{i}} \varepsilon\left(\rho, \chi_{3}\right)\right] \\
& -\frac{\vec{B}}{4 \pi} \cdot \delta_{\chi_{i}}\left(\vec{\nabla} \chi_{1} \times \vec{\nabla} \chi_{2}\right)
\end{aligned}
$$

Now by construction $\vec{v}$ satisfies equation (25) and hence $\frac{\partial \chi_{k}}{\partial t}+\vec{v} \cdot \vec{\nabla} \chi_{k}=0$, this leads to:

$$
\delta_{\chi_{i}} \hat{\mathcal{L}}=-\rho\left[\alpha_{\underline{i}} \frac{d \delta \chi_{\underline{i}}}{d t}+\delta_{\chi_{i}} \varepsilon\left(\rho, \chi_{3}\right)\right]-\frac{\vec{B}}{4 \pi} \cdot \delta_{\chi_{i}}\left(\vec{\nabla} \chi_{1} \times \vec{\nabla} \chi_{2}\right) .
$$

From now on the derivation proceeds as in equations (141517) resulting in equations (1618) and will not be repeated. The difference is that now $\alpha, \beta$ and $\sigma$ are not independent quantities, rather they depend through equation (32) on the derivatives of $\chi_{i}, \nu$. Thus, equations (141517) are not first order equations in time but are second order equations. Now let us calculate the variational derivative with respect to $\nu$ this will result in the expression:

$$
\delta_{\nu} \hat{\mathcal{L}}=-\rho\left[\frac{\partial \delta \nu}{\partial t}+\delta_{\nu} \alpha_{n} \frac{\partial \chi_{n}}{\partial t}\right]
$$

However, $\delta_{\nu} \alpha_{k}$ can be calculated from equation (32):

$$
\delta_{\nu} \alpha_{n}=A_{n j}^{-1} \delta_{\nu} k_{j}=-A_{n j}^{-1} \vec{\nabla} \delta \nu \cdot \vec{\nabla} \chi_{j}
$$

Inserting the above equation into equation (41):

$$
\delta_{\nu} \hat{\mathcal{L}}=-\rho\left[\frac{\partial \delta \nu}{\partial t}-A_{n j}^{-1} \vec{\nabla} \chi_{j} \frac{\partial \chi_{n}}{\partial t} \cdot \vec{\nabla} \delta \nu\right]=-\rho\left[\frac{\partial \delta \nu}{\partial t}+\vec{v} \cdot \vec{\nabla} \delta \nu\right]=-\rho \frac{d \delta \nu}{d t}
$$


The above equation can be put to the form:

$$
\delta_{\nu} \hat{\mathcal{L}}=\delta \nu\left[\frac{\partial \rho}{\partial t}+\vec{\nabla} \cdot(\rho \vec{v})\right]-\frac{\partial(\rho \delta \nu)}{\partial t}-\vec{\nabla} \cdot(\rho \vec{v} \delta \nu)
$$

This obviously leads to the continuity equation (3) and some boundary terms in space and time. The variational derivative with respect to $\rho$ is trivial and the analysis is identical to the one in equation (12) leading to equation (13). To conclude this subsection let us summarize the equations of non-barotropic magnetohydrodynamics:

$$
\begin{aligned}
& \frac{d \nu}{d t}=\frac{1}{2} \vec{v}^{2}-w, \\
& \frac{\partial \rho}{\partial t}+\vec{\nabla} \cdot(\rho \vec{v})=0 \\
& \frac{d \sigma}{d t}=T \\
& \frac{d \alpha}{d t}=\frac{\vec{\nabla} \eta \cdot \vec{J}}{\rho}, \\
& \frac{d \beta}{d t}=-\frac{\vec{\nabla} \chi \cdot \vec{J}}{\rho},
\end{aligned}
$$

in which $\alpha, \beta, \sigma, \vec{v}$ are functionals of $\chi, \eta, s, \nu$ as described above. It is easy to show as in equation (21) that those variational equations are equivalent to the physical equations.

\subsection{Lagrangian and Hamiltonian Densities}

Let us put the Lagrangian density of equation (36) in a slightly more explicit form. First us look at the term $\vec{v}^{2}$ :

$$
\vec{v}^{2}=A_{i j}^{-1} \vec{\nabla} \chi_{i} \frac{\partial \chi_{j}}{\partial t} A_{m n}^{-1} \vec{\nabla} \chi_{m} \frac{\partial \chi_{n}}{\partial t}=A_{i j}^{-1} A_{m n}^{-1} A_{i m} \frac{\partial \chi_{j}}{\partial t} \frac{\partial \chi_{n}}{\partial t}=A_{j n}^{-1} \frac{\partial \chi_{j}}{\partial t} \frac{\partial \chi_{n}}{\partial t}
$$

in the above we use equation (35) and equation (28). Next let us look at the expression:

$$
\begin{aligned}
\alpha_{k}\left[\chi_{i}, \nu\right] \frac{\partial \chi_{k}}{\partial t} & =A_{k j}^{-1} k_{j} \frac{\partial \chi_{k}}{\partial t}=-\left(\frac{\partial \chi_{j}}{\partial t}+\vec{\nabla} \nu \cdot \vec{\nabla} \chi_{j}\right) A_{k j}^{-1} \frac{\partial \chi_{k}}{\partial t} \\
& =-A_{j k}^{-1} \frac{\partial \chi_{j}}{\partial t} \frac{\partial \chi_{k}}{\partial t}-\vec{\nabla} \nu \cdot \vec{\nabla} \chi_{j} A_{k j}^{-1} \frac{\partial \chi_{k}}{\partial t} \\
& =-A_{j k}^{-1} \frac{\partial \chi_{j}}{\partial t} \frac{\partial \chi_{k}}{\partial t}-\frac{\partial \nu}{\partial \chi_{m}} \vec{\nabla} \chi_{m} \cdot \vec{\nabla} \chi_{j} A_{k j}^{-1} \frac{\partial \chi_{k}}{\partial t} \\
& =-A_{j k}^{-1} \frac{\partial \chi_{j}}{\partial t} \frac{\partial \chi_{k}}{\partial t}-\frac{\partial \nu}{\partial \chi_{m}} A_{m j} A_{k j}^{-1} \frac{\partial \chi_{k}}{\partial t} \\
& =-A_{j k}^{-1} \frac{\partial \chi_{j}}{\partial t} \frac{\partial \chi_{k}}{\partial t}-\frac{\partial \nu}{\partial \chi_{m}} \delta_{k m} \frac{\partial \chi_{k}}{\partial t} \\
& =-A_{j k}^{-1} \frac{\partial \chi_{j}}{\partial t} \frac{\partial \chi_{k}}{\partial t}-\frac{\partial \nu}{\partial \chi_{m}} \frac{\partial \chi_{m}}{\partial t}
\end{aligned}
$$


Inserting equation (46) and equation (47) into equation (36) leads to a Lagrangian density of a more standard quadratic form:

$\hat{\mathcal{L}}\left[\chi_{i}, \nu, \rho\right]=\rho\left[\frac{1}{2} A_{j n}^{-1} \frac{\partial \chi_{j}}{\partial t} \frac{\partial \chi_{n}}{\partial t}+\frac{\partial \nu}{\partial \chi_{m}} \frac{\partial \chi_{m}}{\partial t}-\frac{\partial \nu}{\partial t}-\varepsilon\left(\rho, \chi_{3}\right)\right]-\frac{1}{8 \pi}\left(\vec{\nabla} \chi_{1} \times \vec{\nabla} \chi_{2}\right)^{2}$.

In which $A_{j n}^{-1}$ plays the rule of a "metric". The Lagrangian is thus composed of a kinetic terms which is quadratic in the temporal derivatives, a "gyroscopic" terms which is linear in the temporal derivative and a potential term which is independent of the temporal derivative.

In order to obtain a Hamiltonian density of a more convenient form we will add a temporal derivative to the Lagrangian density $\hat{\mathcal{L}}$, this will not change the dynamical equations as is well known, hence we can write:

$$
\begin{aligned}
& \tilde{\mathcal{L}}\left[\chi_{i}, \nu, \rho\right]=\hat{\mathcal{L}}+\frac{\partial(\rho \nu)}{\partial t}= \\
& \nu \frac{\partial \rho}{\partial t}+\rho\left[\frac{1}{2} A_{j n}^{-1} \frac{\partial \chi_{j}}{\partial t} \frac{\partial \chi_{n}}{\partial t}+\frac{\partial \nu}{\partial \chi_{m}} \frac{\partial \chi_{m}}{\partial t}-\varepsilon\left(\rho, \chi_{3}\right)\right]-\frac{1}{8 \pi}\left(\vec{\nabla} \chi_{1} \times \vec{\nabla} \chi_{2}\right)^{2} .
\end{aligned}
$$

From this we obtain the following canonical momenta:

$$
p_{\rho}=\frac{\partial \tilde{\mathcal{L}}}{\partial \dot{\rho}}=\nu, \quad \dot{\rho} \equiv \frac{\partial \rho}{\partial t} .
$$

And:

$$
p_{\chi_{k}}=\frac{\partial \tilde{\mathcal{L}}}{\partial \dot{\chi}_{k}}=\rho\left[A_{k j}^{-1} \frac{\partial \chi_{j}}{\partial t}+\frac{\partial \nu}{\partial \chi_{k}}\right]=-\rho \alpha_{k}, \quad \dot{\chi}_{k} \equiv \frac{\partial \chi_{k}}{\partial t} .
$$

The Hamiltonian density can be now calculated as follows:

$$
\begin{aligned}
\mathcal{H}\left[\chi_{i}, \rho, p_{\chi_{k}}, p_{\rho}\right] & =p_{\chi_{k}} \dot{\chi}_{k}+p_{\rho} \dot{\rho}-\tilde{\mathcal{L}} \\
& =\rho\left[\frac{1}{2} A_{j n}^{-1} \frac{\partial \chi_{j}}{\partial t} \frac{\partial \chi_{n}}{\partial t}+\varepsilon\left(\rho, \chi_{3}\right)\right]+\frac{1}{8 \pi}\left(\vec{\nabla} \chi_{1} \times \vec{\nabla} \chi_{2}\right)^{2} \\
& =\frac{1}{2} \rho^{-1} A_{k l} p_{\chi_{k}} p_{\chi_{l}}-p_{\chi_{k}} \vec{\nabla} \chi_{k} \cdot \vec{\nabla} p_{\rho}+\frac{1}{2} \rho\left(\vec{\nabla} p_{\rho}\right)^{2} \\
& +\rho \varepsilon\left(\rho, \chi_{3}\right)+\frac{1}{8 \pi}\left(\vec{\nabla} \chi_{1} \times \vec{\nabla} \chi_{2}\right)^{2} .
\end{aligned}
$$

Hence by virtue of equation (46) the Hamiltonian density is equal to the energy density of the flow:

$$
\mathcal{H}=\frac{1}{2} \rho \vec{v}^{2}+\rho \varepsilon+\frac{\vec{B}^{2}}{8 \pi}
$$

Hamilton equations are now:

$$
\begin{aligned}
\frac{\delta \mathcal{H}}{\delta p_{\rho}} & =\dot{\rho} \\
-\frac{\delta \mathcal{H}}{\delta \rho} & =\dot{p}_{\rho}
\end{aligned}
$$




$$
\begin{aligned}
\frac{\delta \mathcal{H}}{\delta p_{\chi_{k}}} & =\dot{\chi}_{k} \\
-\frac{\delta \mathcal{H}}{\delta \chi_{k}} & =\dot{p}_{\chi_{k}} .
\end{aligned}
$$

Equation (54) is equivalent to the continuity equation (3). Equation (55) is equivalent to the Bernoulli type equation (13). Equation (56) is equivalent to equation (25) and thus is satisfied identically. Equation (56) is equivalent to equation (16) and equation (18). Hence the above equations are the same as equation (45) but in Hamiltonian presentation.

\section{Conclusion}

It is shown that non-barotropic magnetohydrodynamics can be derived from a variational principle of five functions. The formalism is given in both a Lagrangian and a Hamiltonian presentation.

Possible applications include stability analysis of stationary magnetohydrodynamic configurations and its possible utilization for developing efficient numerical schemes for integrating the magnetohydrodynamic equations. It may be more efficient to incorporate the developed formalism in the frame work of an existing code instead of developing a new code from scratch. Possible existing codes are described in [15, 16, 17, 18, 19. I anticipate applications of this study both to linear and non-linear stability analysis of known barotropic magnetohydrodynamic configurations [20, 21, 22. I suspect that for achieving this we will need to add additional constants of motion constraints to the action as was done by [23, 24] see also 25, 26, 27]. As for designing efficient numerical schemes for integrating the equations of fluid dynamics and magnetohydrodynamics one may follow the approach described in [28, 29, 30, 31].

Another possible application of the variational method is in deducing new analytic solutions for the magnetohydrodynamic equations. Although the equations are notoriously difficult to solve being both partial differential equations and nonlinear, possible solutions can be found in terms of variational variables. An example for this approach is the self gravitating torus described in [32].

One can use continuous symmetries which appear in the variational Lagrangian to derive through Noether theorem new conservation laws. An example for such derivation which still lacks physical interpretation can be found in [35. It may be that the Lagrangian derived in 9 has a larger symmetry group. And of course one anticipates a different symmetry structure for the non-barotropic case.

Topological invariants have always been informative, and there are such invariants in MHD flows. For example the two helicities have long been useful in research into the problem of hydrogen fusion, and in various astrophysical scenarios. In previous works [8, 10, 37] connections between helicities with symmetries of the barotropic fluid equations were made. The variables of the 
current variational principles may help us to identify and characterize as yet unknown topological invariant in MHD.

\section{References}

[1] P. A. Sturrock, Plasma Physics (Cambridge University Press, Cambridge, 1994)

[2] V. A. Vladimirov and H. K. Moffatt, J. Fluid. Mech. 283 125-139 (1995)

[3] A. V. Kats, Los Alamos Archives physics-0212023 (2002), JETP Lett. 77, $657(2003)$

[4] A. V. Kats and V. M. Kontorovich, Low Temp. Phys. 23, 89 (1997)

[5] A. V. Kats, Physica D 152-153, 459 (2001)

[6] T. Sakurai, Pub. Ast. Soc. Japan 31209 (1979)

[7] W. H. Yang, P. A. Sturrock and S. Antiochos, Ap. J., 309383 (1986)

[8] A. Yahalom and D. Lynden-Bell, "Simplified Variational Principles for Barotropic Magnetohydrodynamics," (Los-Alamos Archives physics/0603128) Journal of Fluid Mechanics, Vol. 607, 235-265, 2008.

[9] Yahalom A., " A Four Function Variational Principle for Barotropic Magnetohydrodynamics" EPL 89 (2010) 34005, doi: 10.1209/0295-5075/89/34005 [Los - Alamos Archives - arXiv: 0811.2309]

[10] Asher Yahalom "Aharonov - Bohm Effects in Magnetohydrodynamics" Physics Letters A. Volume 377, Issues 31-33, 30 October 2013, Pages 18981904.

[11] A. Frenkel, E. Levich and L. Stilman Phys. Lett. A 88, p. 461 (1982)

[12] V. E. Zakharov and E. A. Kuznetsov, Usp. Fiz. Nauk 40, 1087 (1997)

[13] J. D. Bekenstein and A. Oron, Physical Review E Volume 62, Number 4, 5594-5602 (2000)

[14] A. V. Kats, Phys. Rev E 69, 046303 (2004)

[15] Mignone, A., Rossi, P., Bodo, G., Ferrari, A., \& Massaglia, S. (2010). Highresolution 3D relativistic MHD simulations of jets. Monthly Notices of the Royal Astronomical Society, 402(1), 7-12.

[16] Miyoshi, T., \& Kusano, K. (2005). A multi-state HLL approximate Riemann solver for ideal magnetohydrodynamics. Journal of Computational Physics, 208(1), 315-344. 
[17] Igumenshchev, I. V., Narayan, R., \& Abramowicz, M. A. (2003). Threedimensional magnetohydrodynamic simulations of radiatively inefficient accretion flows. The Astrophysical Journal, 592(2), 1042.

[18] Faber, J. A., Baumgarte, T. W., Shapiro, S. L., \& Taniguchi, K. (2006). General relativistic binary merger simulations and short gamma-ray bursts. The Astrophysical Journal Letters, 641(2), L93.

[19] Hoyos, J., Reisenegger, A., \& Valdivia, J. A. (2007). Simulation of the Magnetic Field Evolution in Neutron Stars. In VI Reunion Anual Sociedad Chilena de Astronomia (SOCHIAS) (Vol. 1, p. 20).

[20] V. A. Vladimirov, H. K. Moffatt and K. I. Ilin, J. Fluid Mech. 329, 187 (1996); J. Plasma Phys. 57, 89 (1997); J. Fluid Mech. 390, 127 (1999)

[21] Bernstein, I. B., Frieman, E. A., Kruskal, M. D., \& Kulsrud, R. M. (1958). An energy principle for hydromagnetic stability problems. Proceedings of the Royal Society of London. Series A. Mathematical and Physical Sciences, 244(1236), 17-40.

[22] J. A. Almaguer, E. Hameiri, J. Herrera, D. D. Holm, Phys. Fluids, 31, 1930 (1988)

[23] V. I. Arnold, Appl. Math. Mech. 29, 5, 154-163.

[24] V. I. Arnold, Dokl. Acad. Nauk SSSR 162 no. 5.

[25] J. Katz, S. Inagaki, and A. Yahalom, "Energy Principles for SelfGravitating Barotropic Flows: I. General Theory", Pub. Astro. Soc. Japan 45, 421-430 (1993).

[26] Yahalom A., Katz J. \& Inagaki K. 1994, Mon. Not. R. Astron. Soc. 268 506-516.

[27] Asher Yahalom, "Stability in the Weak Variational Principle of Barotropic Flows and Implications for Self-Gravitating Discs". Monthly Notices of the Royal Astronomical Society 418, 401-426 (2011).

[28] A. Yahalom, "Method and System for Numerical Simulation of Fluid Flow", US patent 6,516,292 (2003).

[29] A. Yahalom, \& G. A. Pinhasi, "Simulating Fluid Dynamics using a Variational Principle", proceedings of the AIAA Conference, Reno, USA (2003).

[30] A. Yahalom, G. A. Pinhasi and M. Kopylenko, "A Numerical Model Based on Variational Principle for Airfoil and Wing Aerodynamics", proceedings of the AIAA Conference, Reno, USA (2005).

[31] D. Ophir, A. Yahalom, G.A. Pinhasi and M. Kopylenko " A Combined Variational and Multi-Grid Approach for Fluid Dynamics Simulation" Proceedings of the ICE - Engineering and Computational Mechanics, Volume 165, Issue 1, 01 March 2012, pages 3 -14, ISSN: 1755-0777, E-ISSN: 1755-0785. 
[32] Asher Yahalom "Using fluid variational variables to obtain new analytic solutions of self-gravitating flows with nonzero helicity" Procedia IUTAM 7 (2013) $223-232$.

[33] Clebsch, A., Uber eine allgemeine Transformation der hydro-dynamischen Gleichungen. J. reine angew. Math. 1857, 54, 293-312.

[34] Clebsch, A., Uber die Integration der hydrodynamischen Gleichungen. J. reine angew. Math. 1859, 56, 1-10.

[35] Asher Yahalom, "A New Diffeomorphism Symmetry Group of Magnetohydrodynamics" V. Dobrev (ed.), Lie Theory and Its Applications in Physics: IX International Workshop, Springer Proceedings in Mathematics \& Statistics 36, p. 461-468, 2013.

[36] Katz, J. \& Lynden-Bell, D. Geophysical \& Astrophysical Fluid Dynamics 33,1 (1985).

[37] A. Yahalom, "Helicity Conservation via the Noether Theorem" J. Math. Phys. 36, 1324-1327 (1995). [Los-Alamos Archives solv-int/9407001] 